\title{
Multiple Congenital Anomalies after Assisted Reproductive Technology in Japan (between 2004 and 2009)
}

\author{
Syuichi Ooki \\ Department of Health Science, Ishikawa Prefectural Nursing University, 1-1, Gakuendai, Kahoku, Ishikawa 929-1210, Japan \\ Correspondence should be addressed to Syuichi Ooki; sooki@ishikawa-nu.ac.jp
}

Received 13 August 2012; Accepted 2 October 2012

Academic Editors: J. Lewsey and H. Pollard

Copyright (C) 2013 Syuichi Ooki. This is an open access article distributed under the Creative Commons Attribution License, which permits unrestricted use, distribution, and reproduction in any medium, provided the original work is properly cited.

\begin{abstract}
Multiple congenital anomalies (MCAs) are defined as two or more unrelated major structural malformations that cannot be explained by an underlying syndrome or sequence. The percentage and combination patterns of MCAs were analyzed using the European surveillance of congenital anomalies computer algorithms for nationwide data on congenital anomalies after assisted reproductive technology between 2004 and 2009 in Japan. There were a total of 177,548 pregnancies and 124,846 live births. About $7 \%(96 / 1,324)$ were MCAs. Although most maternal/neonatal outcomes between the isolated cases group and the MCAs group were similar, higher early neonatal death rate was observed in the MCAs group than in the isolated cases group (9.8\% versus 3.7\%, resp.). Regarding the major organ system subcategory in ICD-10, the rate of MCAs was around 30\% in "congenital malformations of eye, ear, face, and neck," "congenital malformations of the respiratory system," and "congenital malformations of genital organs." On the other hand, the rate of MCAs was less than $10 \%$ in "congenital malformations of the circulatory system." The combination patterns of diseases were widely varied. Of them, two or three diseases of the circulatory system, the digestive system, and the musculoskeletal system tended to co-occur in the same individuals.
\end{abstract}

\section{Introduction}

Association among congenital malformations is a concept introduced to designate the nonrandom tendency of some malformations to occur together more often than expected by chance, without being components of known syndromes [1]. The utility of monitoring for patterns of multiple congenital anomalies (MCAs) has been increasingly recognized [2-6]. The possible reasons for this association are the results of known syndromes, as a consequence of a single primary anomaly, and known or unknown factors causing MCAs. This kind of coexistence is called comorbidity in general, and it is important to clarify the genetic/environmental background of associated malformations. According to Garne et al. [7], MCAs are defined as two or more unrelated major structural malformations that cannot be explained by an underlying syndrome or sequence.

Since etiologic heterogeneity may complicate epidemiologic analyses designed to identify risk factors for congenital anomalies, case classification, which uses knowledge of embryologic and pathogenic mechanisms [8], is important to make case groups more homogeneous [9]. Wellesley et al. [10] produced a hierarchical method of classifying congenital anomalies into eight groups for inclusion of the source of data registration in conjunction with, but independent of, International Classification of Disease (ICD) coding. The eight disease categories are chromosome, microdeletion, teratogen, new dominant, familial, syndromes, isolated, and multiple. Thus, there is fairly agreement in the literature on a gross etiologic classification of congenital anomalies [911] for surveillance and epidemiologic studies to be used in combination with an anatomic approach based on organ system or an approach based on presumed pathogenesis [7]. The proposed classifications contain the following categories: chromosomal syndromes, monogenic syndromes, environmental syndromes, isolated congenital anomalies, and MCAs.

Surveillance of MCAs is considered to be more sensitive for the detection of new teratogens than is surveillance of all or isolated congenital anomalies [7, 12]. The European 
surveillance of congenital anomalies (EUROCAT [13]) is a network of European registers of congenital anomalies carrying out epidemiologic surveillance of congenital anomalies, and it currently covers a population of 1.7 million births per year. Recently, the EUROCAT published a computer algorithm for the classification of major congenital anomaly cases ([7] see Methods).

The purpose of the present study was to estimate the frequency of MCAs and further examine their characteristics by adapting the EUROCAT computer algorithm and applying it to nationwide data on the congenital anomalies in Japan.

\section{Materials and Methods}

\subsection{Outline of Japanese Birth Defects Data after Assisted} Reproductive Technology (ART). The method for collecting data is described in detail elsewhere $[14,15]$. Almost all medical institutions performing ART are registered with the Japan Society of Obstetrics and Gynecology (JSOG), which administers questionnaire surveys for these medical institutions. Some of the survey data are presented in simple annual reports of aggregate, not individual, data. From 2004 to 2009 (the latest), the individual list of all ART pregnancies resulting in congenital anomalies was presented every year in the JSOG annual reports on ART (in Japanese) with an almost $100 \%$ response rate. The author used these case report data as initial information for the present existing data analyses.

These case reports were classified according to the computer algorithm developed by the EUROCAT [7], which was based on the International Classification of Disease version 10 (ICD-10) codes. This algorithm gives explicit coding rules for classification and picks out a small subset of cases of potential MCAs. The etiologic classification was translated into a computer algorithm that imposes a hierarchical classification according to the ICD codes given for each case [7]. The outline of the computer algorithm is as follows: the first category is "chromosomal," to which a case is allocated if it has a chromosomal ICD code, namely, Q90-Q93 (except Q936) or Q96-Q99. Only cases that are not allocated to "chromosomal" are considered for the next step. The next category is the "genetic syndromes," "skeletal dysplasia," and "congenital skin disorder" codes. The next code is the "teratogenic syndrome" code. Thus, after 21 steps are performed, any remaining case is allocated to the "potential multiple anomaly" category only if it does not belong to any of the preceding categories. All cases are classified into the following four main categories: chromosomal case, syndromes, isolated single anomalies, and "potential" MCAs. Although the EUROCAT further checked the "potential" multiples to determine whether these cases were "true" MCAs by three clinical geneticists [7], this procedure is beyond the present descriptive epidemiologic study.

According to the EUROCAT definition, cases of congenital anomalies for live births and fetal deaths with gestational age $\geq 20$ weeks were selected. In total, 1,263 live births and 61 stillbirths were analyzed in the present study. The number of total ART live births were 124,846 between 2004 and 2009. The number of stillbirths ( $\geq 22$ weeks, according to the definition of the JSOG report) was not reported in the
JSOG data. The number of stillbirths deliveries (numbers of mothers) was 747 in the same period.

2.2. Statistical Analysis. First, all cases were classified into the four main categories according to the EUROCAT algorithm. Then, demographic characteristics between the isolated cases group and the MCAs group were compared. For the comparison between the isolated cases group and the MCAs group, the $t$-test was performed for maternal age and gestational weeks and the $\chi^{2}$ test for method of fertility treatment, blastocyst transfer, plurality, sex, and perinatal outcome. The significance level was set at .05. The proportions of cases classified as isolated anomalies, syndromes (chromosomal, monogenic, and environmental), and MCAs by the ICD10 main organ system subcategories were analyzed. Finally, typical combination patterns of two ICD-10 subcategories in MCAs were examined. If certain patients had more than two diseases from different ICD-10 subcategories, for example, diseases "A, B, and C", these diseases were broken down as several combinations of two diseases, "A and B," "A and C," and "B and C."

Newborn conditions and those of prematurity were not considered MCAs. A higher proportion of patent ductus arteriosus (PDA, Q250) in multiple births is frequently observed [16-18] and is attributed to the prematurity or shorter gestational age of multiples [17, 19]. Since PDA was also frequently seen among multiple births in the present data [15], the analyses were performed both with and without PDA.

\section{Results}

The results of the case classification by the EUROCAT algorithm are shown in Table 1. Two hundred and nine cases with more than one disease were classified into 68 isolated single anomalies, 36 chromosomal cases, 9 syndromes, and 96 potential MCAs. Thus, final classification of the 1,324 cases was as follows: 203 chromosomal cases (15.3\%), 45 syndromes (3.4\%), 980 (74.0\%) isolated single anomalies, and $96(7.3 \%)$ potential MCAs. About half of the cases with more than one disease were reclassified into other categories than potential MCAs. This result was nearly the same as that obtained for the EUROCAT registries, although the present results were "potential" MCAs. About 17\% (16/96) of MCAs included more than two anomalies.

The perinatal characteristics of the isolated cases group and the MCAs group are shown in Table 2. The frequency of early neonatal death was significantly higher among the MCAs group compared to the isolated cases group (9.8\% versus $3.7 \%$, resp.). There was no difference with regard to method of fertility treatment, blastocyst transfer, maternal age, gestational weeks, plurality, sex, or percentage of stillbirths between the isolated cases group and the MCAs group.

The proportions of cases classified as isolated anomalies, syndromes (chromosomal, monogenic, and environmental), and MCAs are shown according to ICD-10 major organ system categories in Table 3. The rate of MCAs was around $30 \%$ in "congenital malformations of eye, ear, face, and neck (Q10-Q18)," "congenital malformations of the respiratory 


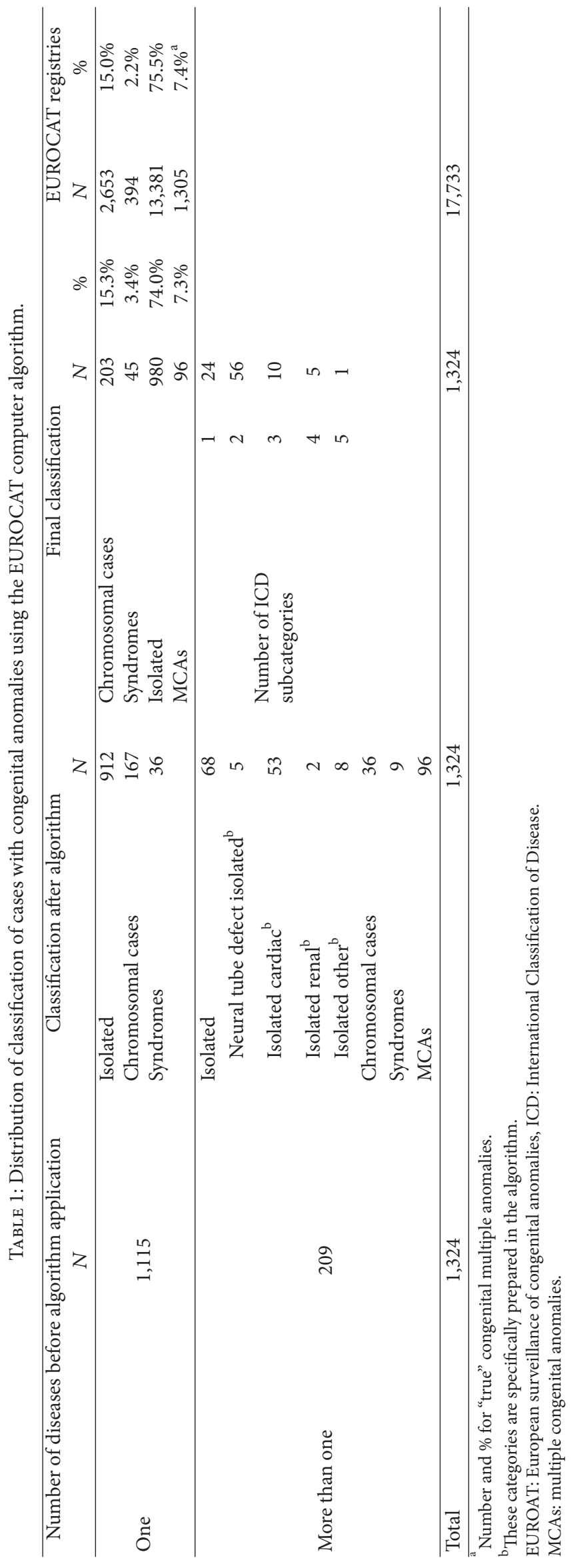


TABLE 2: Demographic and perinatal outcome between isolated and multiple congenital anomalies.

\begin{tabular}{|c|c|c|c|c|c|}
\hline & \multicolumn{2}{|c|}{$\begin{array}{c}\text { Isolated } \\
(N=980)\end{array}$} & \multicolumn{2}{|c|}{$\begin{array}{c}\text { MCAs } \\
(N=96)\end{array}$} & \multirow[t]{2}{*}{$P$} \\
\hline & $N$ & $\%$ & $N$ & $\%$ & \\
\hline \multicolumn{6}{|l|}{ Method of fertility treatment } \\
\hline IVF & 239 & 24.4 & 25 & 26.0 & \\
\hline ICSI & 236 & 24.1 & 22 & 22.9 & \\
\hline IVF and ICSI & 40 & 4.1 & 5 & 5.2 & \\
\hline Frozen embryo transfer & 463 & 47.2 & 44 & 45.8 & $0.930^{\mathrm{a}}$ \\
\hline Unknown/missing values & 2 & 0.2 & 0 & 0.0 & \\
\hline \multicolumn{6}{|l|}{ Blastocyst transfer } \\
\hline Yes & 507 & 51.7 & 48 & 50.0 & \\
\hline No & 464 & 47.3 & 48 & 50.0 & $0.679^{\mathrm{a}}$ \\
\hline Unknown/missing values & 9 & 0.9 & 0 & 0.0 & \\
\hline \multicolumn{6}{|l|}{ Maternal age } \\
\hline Range & \multicolumn{2}{|c|}{$23-45$} & \multicolumn{2}{|c|}{$24-42$} & \\
\hline Mean \pm SD & \multicolumn{2}{|c|}{$34.3 \pm 3.9$} & \multicolumn{2}{|c|}{$34.0 \pm 3.8$} & $0.459^{\mathrm{b}}$ \\
\hline Median & \multicolumn{2}{|c|}{34} & \multicolumn{2}{|c|}{34} & \\
\hline \multicolumn{6}{|l|}{ Gestational weeks } \\
\hline Range & \multicolumn{2}{|c|}{$20-42$} & \multicolumn{2}{|c|}{$21-41$} & \\
\hline Mean \pm SD & \multicolumn{2}{|c|}{$37.2 \pm 3.7$} & \multicolumn{2}{|c|}{$36.7 \pm 3.9$} & $0.234^{\mathrm{b}}$ \\
\hline Median & \multicolumn{2}{|c|}{38} & \multicolumn{2}{|c|}{37} & \\
\hline Unknown/missing values & \multicolumn{2}{|c|}{19} & \multicolumn{2}{|c|}{2} & \\
\hline \multicolumn{6}{|l|}{ Plurality } \\
\hline Singletons & 791 & 80.7 & 73 & 76.0 & \\
\hline Multiple births & 188 & 19.2 & 21 & 21.9 & $0.463^{\mathrm{a}}$ \\
\hline Unknown/missing values & 1 & 0.1 & 2 & 2.1 & \\
\hline \multicolumn{6}{|l|}{ Sex (fetuses/neonates) } \\
\hline Male & 547 & 55.8 & 52 & 54.2 & \\
\hline Female & 409 & 41.7 & 37 & 38.5 & $0.825^{\mathrm{a}}$ \\
\hline Unknown/missing values & 24 & 2.4 & 7 & 7.3 & \\
\hline \multicolumn{6}{|c|}{ Perinatal outcome (fetuses/neonates) } \\
\hline Stillbirths $(20 \geq$ weeks $)$ & 25 & 2.6 & 4 & 4.2 & \\
\hline Live births & 955 & 97.4 & 92 & 95.8 & $0.351^{\mathrm{a}}$ \\
\hline Unknown/missing values & 0 & 0.0 & 0 & 0.0 & \\
\hline \multicolumn{6}{|c|}{ Early neonatal death (neonatal death up to day 6 after birth) } \\
\hline Yes & 35 & 3.7 & 9 & 9.8 & \\
\hline No & 687 & 71.9 & 63 & 68.5 & $0.007^{\mathrm{a}}$ \\
\hline Unknown/missing values & 233 & 24.4 & 20 & 21.7 & \\
\hline
\end{tabular}

MCAs: multiple congenital anomalies, IVF: in vitro fertilization, ICSI: intracytoplasmic sperm injection.

n.s.: not significant.

${ }^{\mathrm{a}} \chi^{2}$ test, ${ }^{\mathrm{b}} t$-test. Unknown/missing values were excluded from the statistical tests.

system (Q30-Q34)," and "congenital malformations of genital organs (Q50-Q56)." On the other hand, rate of MCAs was $8 \%$ in "congenital malformations of the circulatory system (Q20-Q28)."

The patterns of association of ICD-10 subcategory in MCAs are shown in Table 4 . Twenty-four cases (25\%) had more than one disease within the same ICD-10 subcategory. "Congenital malformations of the circulatory system (Q20-Q28)," "other congenital malformations of the digestive system (Q38-Q45)," and "congenital malformations and deformations of the musculoskeletal system (Q65-Q79)" tended to co-occur. "Cleft lip and cleft palate (Q35-Q37)" also tended to be associated with other congenital anomalies. Forty-four different patterns of combination at the ICD-10 organ system subcategory level were detected for 96 MCAs. But the diseases co-occurrence at a more detailed disease classification level, namely, the two-digit code after Q (e.g., Q21 for congenital malformations of cardiac septa), was rarely observed. Eighty-four cases out of 96 MCAs (88\%) had different combination patterns at the two-digit code after $\mathrm{Q}$ 
TABLE 3: Proportions of isolated anomalies, syndromes (chromosomal, monogenic, and environmental), and MCAs according to the ICD-10 major organ system categories.

\begin{tabular}{|c|c|c|c|c|c|c|c|}
\hline \multirow{2}{*}{ ICD-10 } & & \multicolumn{2}{|c|}{ Isolated } & \multicolumn{2}{|c|}{ Syndromes } & \multicolumn{2}{|c|}{ MCAs } \\
\hline & & $N$ & $\%$ & $N$ & $\%$ & $N$ & $\%$ \\
\hline Q00-Q07 & Congenital malformations of the nervous system & 63 & 79.7 & 2 & 2.5 & 14 & 17.7 \\
\hline Q10-Q18 & Congenital malformations of eye, ear, face, and neck & 61 & 70.9 & 2 & 2.3 & 23 & 26.7 \\
\hline Q20-Q28 & Congenital malformations of the circulatory system & 399 & 85.4 & 30 & 6.4 & 38 & 8.1 \\
\hline Q30-Q34 & Congenital malformations of the respiratory system & 14 & 66.7 & 0 & 0.0 & 7 & 33.3 \\
\hline Q35-Q37 & Cleft lip and cleft palate & 81 & 81.8 & 2 & 2.0 & 16 & 16.2 \\
\hline Q38-Q45 & Other congenital malformations of the digestive system & 93 & 73.2 & 10 & 7.9 & 24 & 18.9 \\
\hline Q50-Q56 & Congenital malformations of genital organs & 41 & 71.9 & 1 & 1.8 & 15 & 26.3 \\
\hline Q60-Q64 & Congenital malformations of the urinary system & 42 & 77.8 & 0 & 0.0 & 12 & 22.2 \\
\hline Q65-Q79 & Congenital malformations and deformations of the musculoskeletal system & 167 & 77.0 & 12 & 5.5 & 38 & 17.5 \\
\hline Q80-Q89 & Other congenital malformations & 19 & 30.6 & 39 & 62.9 & 4 & 6.5 \\
\hline
\end{tabular}

ICD: International Classification of Disease, MCAs: multiple congenital anomalies.

The cases with more than one disease name (syndromes and MCAs) were counted in several ICD subgroups according to the disease category number.

TABLE 4: Combination patterns in MCAs.

\begin{tabular}{|c|c|c|}
\hline Pattern of combination & $N$ & $\%$ \\
\hline \multicolumn{3}{|c|}{ Combination within the same subcategory } \\
\hline Q10-Q18 & 6 & 6.3 \\
\hline Q65-Q79 & 9 & 9.4 \\
\hline Subtotal & 24 & 25.0 \\
\hline \multicolumn{3}{|c|}{$\begin{array}{c}\text { Combination among different subcategories } \\
\text { (multiple counts allowed) }\end{array}$} \\
\hline Q10-Q18 and Q20-Q28 & 7 & 7.3 \\
\hline Q10-Q18 and Q35-Q37 & 5 & 5.2 \\
\hline Q10-Q18 and Q65-Q79 & 6 & 6.3 \\
\hline Q20-Q28 and Q35-Q37 & 6 & 6.3 \\
\hline Q20-Q28 and Q38-Q45 & 10 & 10.4 \\
\hline Q20-Q28 and Q65-Q79 & 10 & 10.4 \\
\hline Q35-Q37 and Q65-Q79 & 5 & 5.2 \\
\hline Q38-Q45 and Q65-Q79 & 12 & 12.5 \\
\hline
\end{tabular}

MCAs: multiple congenital anomalies.

For an explanation of ICD-10 Q codes, see Table 3.

Percentage was calculated for the total MCAs $(n=96)$.

level. The combination pattern occurring in three or more cases across organ system subcategory were the following: Q17 (other congenital malformations of ear) and Q21 $(n=$ 4), Q21 and Q35 (cleft palate) $(n=3)$, and Q21 and Q37 (cleft palate with cleft lip) $(n=3)$.

There were 7 MCAs cases that included PDA (Q250). If PDA was excluded, three cases transferred to the isolated cases group. Thus, the percentage of potential MCAs became $7.0 \%(93 / 1,324)$. The other four cases contained more than two anomalies.

\section{Discussion}

The main aim of this study was to show descriptive epidemiologic/statistic data on the MCAs using nationwide data and not to refer to the pathogenesis of MCAs. The subjects of this study were all live births/stillbirths after ART. Concerning the comparison of congenital anomalies between spontaneous and ART, larger studies have suggested that children born after ART have an increased risk of congenital anomalies compared with children conceived spontaneously [20]. Data from meta-analyses consistently suggest that the overall risk of major birth defects in children born after ART is about 30\% higher than in children conceived spontaneously [21, 22]. A nationwide survey in Sweden also showed a slightly increased risk for congenital anomalies after IVF (in vitro fertilization), even adjusting for possible confounding factors, such as year of birth, maternal age, and parity [23]. To my knowledge, there was no population/registry-based study examining ART and MCAs. In other words, the present study sheds light on the necessity for this kind of study.

The percentage of potential MCAs was around 7\% in the present subjects. The present distribution of percentage of the four main categories classified using the EUROCAT algorithm was almost the same as those of European registries (Table 1), although the present percentage of MCAs included potential false-positive cases, not necessarily true cases. If more frequent and improved chromosomal analysis and molecular testing become widely used, the percentage of any chromosomal abnormalities/syndromes would become higher.

Given the complicated nature and severity of MCAs, higher early neonatal death observed in the MCAs group than in the isolated cases group seemed reasonable. The percentage of multiple births among the present subjects was obviously higher than that of the general population due to the effect of ART [24]. In addition, the prevalence of congenital anomalies in multiple births is higher than that of singletons [17, 18, 25-27]. There was no difference in the percentage of MCAs between singletons and multiple births in the present study. One possible reason was that multiple births after ART usually produce dizygotic twins, and their pre- and perinatal outcome, including the prevalence of congenital anomalies, is better than that of monozygotic twins [28]. Unfortunately, the present data have no information on the 
correct zygosity for the same-sexed twin pairs. Therefore, the higher proportion of congenital anomalies in multiple births compared to singletons frequently seen in spontaneous pregnancies or total pregnancies may be diminished in ART pregnancies.

The percentage of MCAs according to ICD subcategory and their combination patterns for several specific congenital anomalies were also analyzed. According to the recent systematic population-based study by Miller et al. [29], among 7,984 live-born and stillborn infants and fetuses with congenital heart diseases (CHDs), 71.3\% had isolated anomalies, $13.5 \%$ had MCAs, $13.1 \%$ had syndromes, and $2.0 \%$ had laterality defects. The rate of CHDs in other population-based epidemiologic studies ranged from $6.5 \%$ to $14.1 \%$ [30-33]. The present percentage (8.1\%) of MCAs was well within this range. Contrary to the high prevalence of CHDs, multiple cases occupied a small percentage compared to the percentage of MCAs in other ICD categories, as shown in Table 3.

In a study in a tertiary hospital for the treatment of oral cleft patients, Barbosa et al. [34] reported that 21 patients had CHDs among 220 child patients with cleft lip and/or cleft palate (9.5\%). In the present subjects, 6.9\% (6/87) of subjects with cleft lip and/or cleft palate had any CHD (not shown in Table).

Two or three diseases of the circulatory system (Q20Q28), the digestive system (Q38-Q45), and the musculoskeletal system (Q65-Q79) tended to co-occur in the same individual, as shown in Table 4. According to Miller et al. [29], for cases with any CHDs as a group, the most common noncardiac defects were any skeletal defects (35.0\%), followed closely by gastrointestinal $(25.2 \%)$ and renal defects (23.1\%). Some of these associations might be explained by a common pathogenic mechanism, that is, early differentiation and migration of mesodermal cells for cardiac and skeletal disease or disturbances of neural crest cell migration for cardiac and gastrointestinal disease [29].

Most of these combinations, which occurred at the main organ system level, however, disappeared at a more detailed combination of anomaly level in each subcategory, suggesting the complicated causes of MCAs. The wide variation in the proportion and type of MCAs reported has been due mainly to differences in the types of case (whether based on autopsy report, clinical study, or epidemiologic study) and defect classifications [29]. Moreover, there is some debate about whether individual defect types, isolated or MCAs, represent etiologically distinct groupings [7]. According to Khoury et al. [12], expanding the definition of MCAs from cases with three or more defects to cases with two or more defects not only increases the reporting case load but also includes in the database some infants who have the two defects in question on the basis of chance alone. If combinations of defects must be monitored, then the number of possibilities increases greatly. The number of biologically possible combinations would be lower, and a compromise between splitting and lumping of diseases combination must be achieved [12]. Therefore, a decision must be made on a list of defects to monitor together with their combination to minimize the problems mentioned above.
The statistical methods of classifying MCAs have progressed [1, 11, 12, 35-37]. Nevertheless, a lower prevalence of each MCA makes sophisticated statistical analysis difficult. A population-based twin study using multivariate genetic analyses based on structural equation modeling is one of the most powerful tools to clarify the genetic/environmental contribution to the comorbidity of the diseases [38], including congenital anomalies. Most registries of congenital anomalies do not seem to have precise information on the twin or multiple births cases, including information on concordance/discordance of pairs, sex combination of pairs, and their zygosity. Record linkage between congenital anomalies, including information on the MCAs, and twin registry would make effective statistical genetic analysis possible.

This study has the following limitations, most of which could be attributed to the dataset and due to the fact that individual information was obtained only from the subjects with congenital anomalies. The first and greatest limitation is that the author could not check the reliability of the data directly. This is the essential limitation of existing data analysis. Second, the author could not control for confounding factors that can affect ART and/or congenital anomalies, such as maternal age, parity, smoking, socioeconomic status, medical history, and prenatal care. However, to date, many studies have not necessarily controlled for confounding factors. Third, followup after birth was limited to the neonatal period at the latest and was incomplete. Some congenital anomalies are not obvious within a few days after birth.

\section{Conclusions}

MCAs comprised about 7\% $(96 / 1,324)$ of cases among multiyear nationwide data on congenital anomalies after ART in Japan. The combination patterns of diseases were widely varied. Of them, two or three diseases that belong to the circulatory system, the digestive system and the musculoskeletal system tended to co-occur in the same individual. Future research based on the standardizing populations and methods of analysis is needed.

\section{Acknowledgments}

The author would like to thank Toshimi Ooma for assistance with data analysis. This work was supported in part by a Grant-in-Aid for Challenging Exploratory Research from the Japan Society for the Promotion of Science (Grant no. 23659356).

\section{References}

[1] K. Källén, P. Mastroiacovo, E. E. Castilla, E. Robert, and B. Källén, "VATER non-random association of congenital malformations: study based on data from four malformation registers," American Journal of Medical Genetics, vol. 101, no. 1, pp. 26-32, 2001.

[2] E. E. Castilla, I. M. Orioli, and J. S. Lopez-Camelo, "On monitoring the multiply malformed infant. I: case-finding, caserecording, and data handling in a Latin American program," American Journal of Medical Genetics, vol. 22, no. 4, pp. 717-725, 1985. 
[3] A. Czeizel, P. Kiss, M. Osztovics, and I. Pazonyi, "Nationwide investigation of multiple malformations," Acta Paediatrica Academiae Scientiarum Hungaricae, vol. 19, no. 4, pp. 275-280, 1978.

[4] B. Källén and J. Winberg, "Multiple malformations studied with a national register of malformations," Pediatrics, vol. 44, no. 3 , pp. 410-417, 1969.

[5] B. Källén, "Population surveillance of multimalformed infants-experience with the Swedish registry of congenital malformations. First part," Journal de Genetique Humaine, vol. 35, no. 4, pp. 205-215, 1987.

[6] M. J. Khoury, M. M. Adams, P. Rhodes, and J. D. Erickson, "Monitoring for multiple malformations in the detection of epidemics of birth defects," Teratology, vol. 36, no. 3, pp. 345-353, 1987.

[7] E. Garne, H. Dolk, M. Loane et al., "Paper 5: surveillance of multiple congenital anomalies: implementation of a computer algorithm in European registers for classification of cases," Birth Defects Research A, vol. 91, no. 1, pp. S44-S50, 2011.

[8] M. L. Martínez-Frías, "Editorial comment: approaches to the analysis of infants with multiple congenital anomalies," American Journal of Medical Genetics, vol. 101, no. 1, pp. 33-35, 2001.

[9] S. A. Rasmussen, R. S. Olney, L. B. Holmes, A. E. Lin, K. M. Keppler-Noreuil, and C. A. Moore, "Guidelines for case classification for the National Birth Defects Prevention Study," Birth Defects Research A, vol. 67, no. 3, pp. 193-201, 2003.

[10] D. Wellesley, P. Boyd, H. Dolk, and S. Pattenden, "An aetiological classification of birth defects for epidemiological research," Journal of Medical Genetics, vol. 42, no. 1, pp. 54-57, 2005.

[11] J. M. Friedman, "The use of dysmorphology in birth defects epidemiology," Teratology, vol. 45, no. 2, pp. 187-193, 1992.

[12] M. J. Khoury, L. Botto, P. Mastroiacovo, R. Skjaerven, E. Castilla, and J. D. Erickson, "Monitoring for multiple congenital anomalies: an international perspective," Epidemiologic Reviews, vol. 16, no. 2, pp. 335-350, 1994.

[13] P. A. Boyd, M. Haeusler, I. Barisic, M. Loane, E. Garne, and H. Dolk, "Paper 1: the EUROCAT network-organization and processes," Birth Defects Research A, vol. 91, no. 1, pp. S2-S15, 2011.

[14] S. Ooki, "Birth defects in singleton versus multiple ART births in Japan (2004-2008)," Journal of Pregnancy, vol. 2011, Article ID 285706, 8 pages, 2011.

[15] S. Ooki, "Birth defects after assisted reproductive technology in Japan: comparison between multiples and singletons, 2004-2009," Reproductive System \& Sexual Disorders, S5, article $3,2012$.

[16] P. M. Layde, J. D. Erickson, A. Falek, and B. J. McCarthy, "Congenital malformation in twins," American Journal of Human Genetics, vol. 32, no. 1, pp. 69-78, 1980.

[17] S. J. Li, N. Ford, K. Meister, and J. Bodurtha, "Increased risk of birth defects among children from multiple births," Birth Defects Research A, vol. 67, no. 10, pp. 879-885, 2003.

[18] Y. Tang, C. X. Ma, W. Cui et al., "The risk of birth defects in multiple births: a population-based study," Maternal and Child Health Journal, vol. 10, no. 1, pp. 75-81, 2006.

[19] A. Pinborg, A. Loft, and A. N. Andersen, "Neonatal outcome in a Danish national cohort of 8602 children born after in vitro fertilization or intracytoplasmic sperm injection: the role of twin pregnancy," Acta Obstetricia et Gynecologica Scandinavica, vol. 83, no. 11, pp. 1071-1078, 2004.
[20] C. Williams, A. Sutcliffe, and N. J. Sebire, "Congenital malformations after assisted reproduction: risks and implications for prenatal diagnosis and fetal medicine," Ultrasound in Obstetrics and Gynecology, vol. 35, no. 3, pp. 255-259, 2010.

[21] A. A. Rimm, A. C. Katayama, M. Diaz, and K. P. Katayama, "A meta-analysis of controlled studies comparing major malformation rates in IVF and ICSI infants with naturally conceived children," Journal of Assisted Reproduction and Genetics, vol. 21, no. 12, pp. 437-443, 2004.

[22] M. Hansen, C. Bower, E. Milne, N. de Klerk, and J. J. Kurinczuk, "Assisted reproductive technologies and the risk of birth defects-a systematic review," Human Reproduction, vol. 20, no. 2, pp. 328-338, 2005.

[23] B. Källén, O. Finnström, A. Lindam, E. Nilsson, K. G. Nygren, and P. O. Otterblad, "Congenital malformations in infants born after in vitro fertilization in Sweden," Birth Defects Research A, vol. 88, no. 3, pp. 137-143, 2010.

[24] S. Ooki, "Effect of maternal age and fertility treatment on the increase in multiple births in Japan: vital Statistics, 1974-2009," Journal of Epidemiology, vol. 21, no. 6, pp. 507-511, 2011.

[25] S. V. Glinianaia, J. Rankin, and C. Wright, "Congenital anomalies in twins: a register-based study," Human Reproduction, vol. 23, no. 6, pp. 1306-1311, 2008.

[26] X. H. Zhang, L. Q. Qiu, and J. P. Huang, "Risk of birth defects increased in multiple births," Birth Defects Research A, vol. 91, no. 1, pp. 34-38, 2011.

[27] P. Mastroiacovo, E. E. Castilla, C. Arpino et al., "Congenital malformations in twins: an international study," American Journal of Medical Genetics, vol. 83, no. 2, pp. 117-124, 1999.

[28] J. Joy, N. McClure, and I. E. Cooke, "A comparison of spontaneously conceived twins and twins conceived by artificial reproductive technologies," Journal of Obstetrics and Gynaecology, vol. 28, no. 6, pp. 580-585, 2008.

[29] A. Miller, T. Riehle-Colarusso, C. J. Alverson, J. L. Frías, and A. Correa, "Congenital heart defects and major structural noncardiac anomalies, Atlanta, Georgia, 1968 to 2005," Journal of Pediatrics, vol. 159, no. 1, pp. 70.e2-78.e2, 2011.

[30] P. Pradat, "Noncardiac malformations at major congenital heart defects," Pediatric Cardiology, vol. 18, no. 1, pp. 11-18, 1997.

[31] V. Grech and M. Gatt, "Syndromes and malformations associated with congenital heart disease in a population-based study," International Journal of Cardiology, vol. 68, no. 2, pp. 151-156, 1999.

[32] A. Meberg, J. Hals, and E. Thaulow, "Congenital heart defects-chromosomal anomalies, syndromes and extracardiac malformations," Acta Paediatrica, vol. 96, no. 8, pp. 1142-1145, 2007.

[33] E. Calzolari, G. Garani, G. Cocchi et al., "Congenital heart defects: 15 Years of experience of the Emilia-Romagna Registry (Italy)," European Journal of Epidemiology, vol. 18, no. 8, pp. 773-780, 2003.

[34] M. M. Barbosa, C. M. G. Rocha, T. Katina, M. Caldas, A. Codorniz, and C. Medeiros, "Prevalence of congenital heart diseases in oral cleft patients," Pediatric Cardiology, vol. 24, no. 4, pp. 369-374, 2003.

[35] M. J. Khoury, L. M. James, and J. D. Erickson, "On the measurement and interpretation of birth defect associations in epidemiologic studies," American Journal of Medical Genetics, vol. 37, no. 2, pp. 229-236, 1990.

[36] T. H. Beaty, P. Yang, M. J. Khoury, E. L. Harris, and K. Y. Liang, "Using log-linear models to test for associations 
among congenital malformations," American Journal of Medical Genetics, vol. 39, no. 3, pp. 299-306, 1991.

[37] K. B. M. Källén, E. E. Castilla, M. Da Graça Dutra, P. Mastroiacovo, E. Robert, and B. A. J. Källén, "A modified method for the epidemiological analysis of registry data on infants with multiple malformations," International Journal of Epidemiology, vol. 28, no. 4, pp. 701-710, 1999.

[38] M. C. Neale and L. R. Cardon, Methodology for Genetic Studies of Twins and Families, International Journal of Epidemiology, Kluwer Academic, Dordrecht, The Netherlands, 1992. 


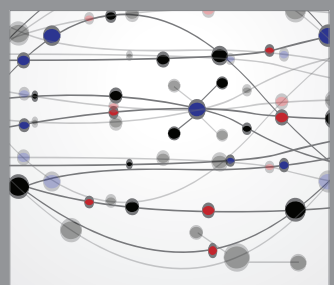

The Scientific World Journal
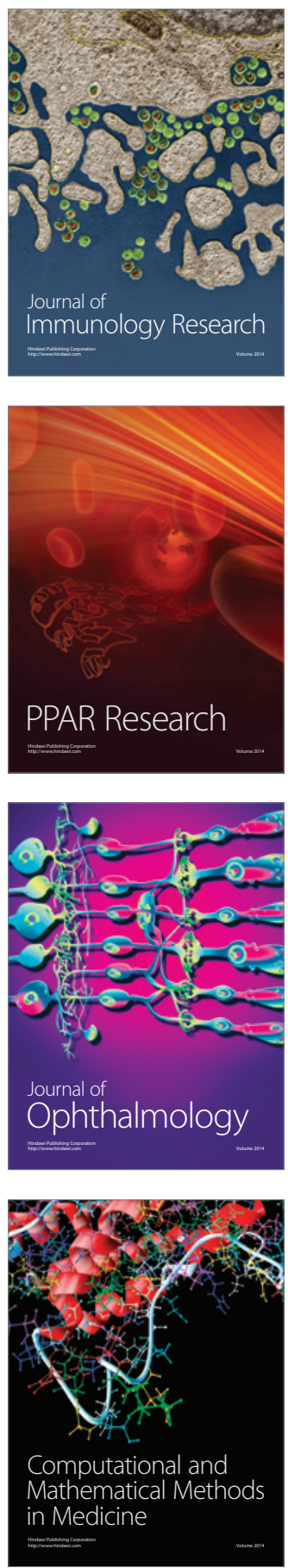

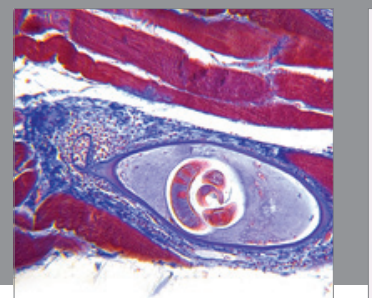

Gastroenterology

Research and Practice
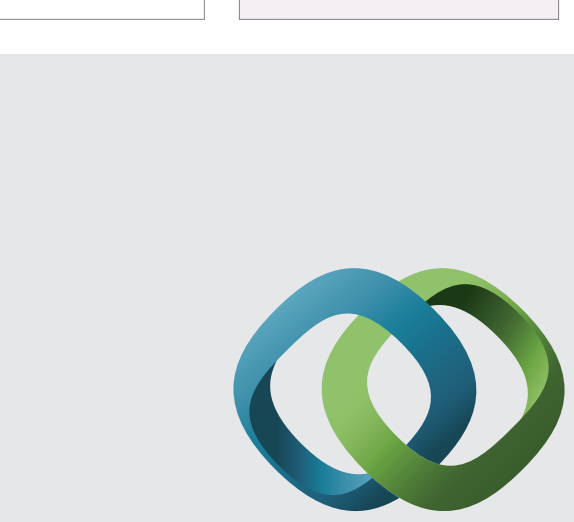

\section{Hindawi}

Submit your manuscripts at

http://www.hindawi.com
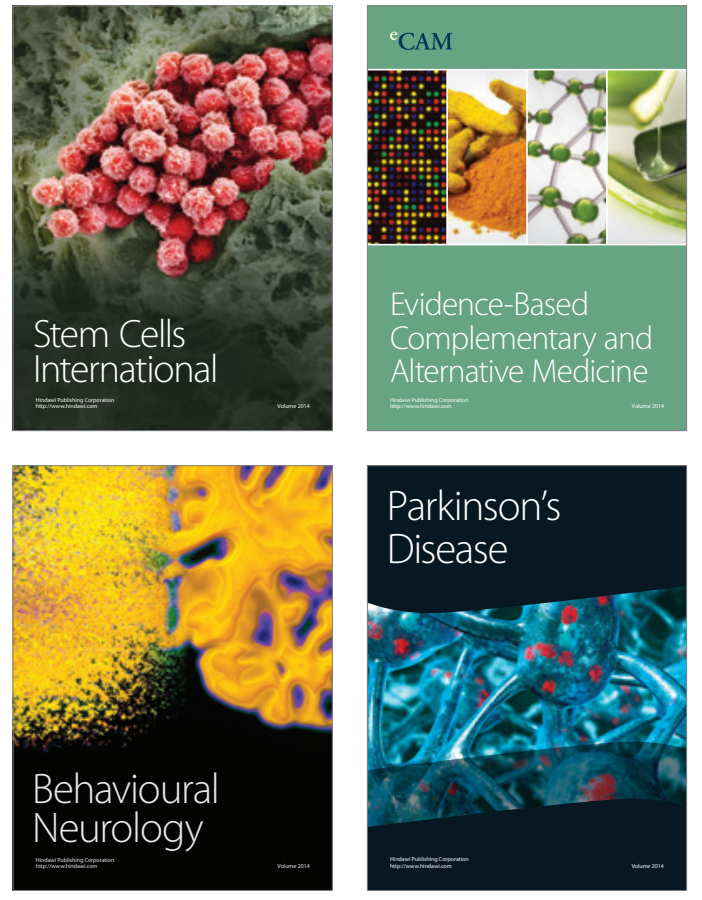
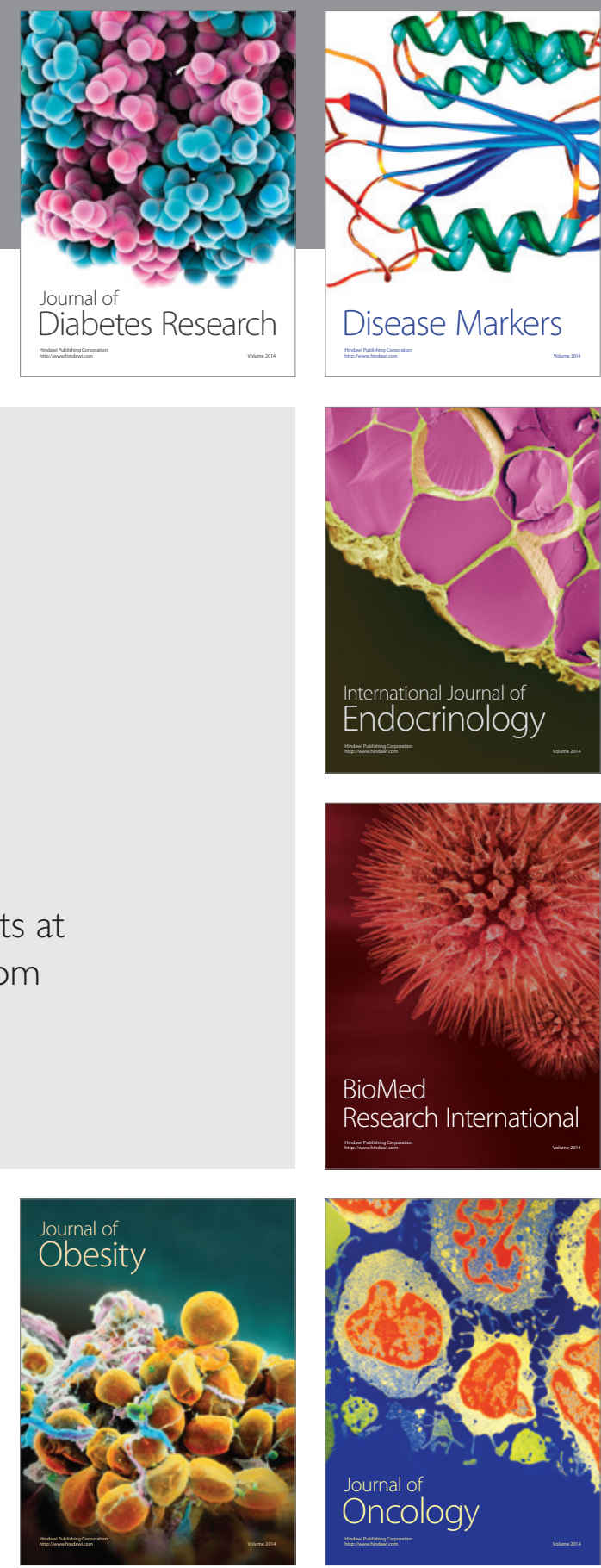

Disease Markers
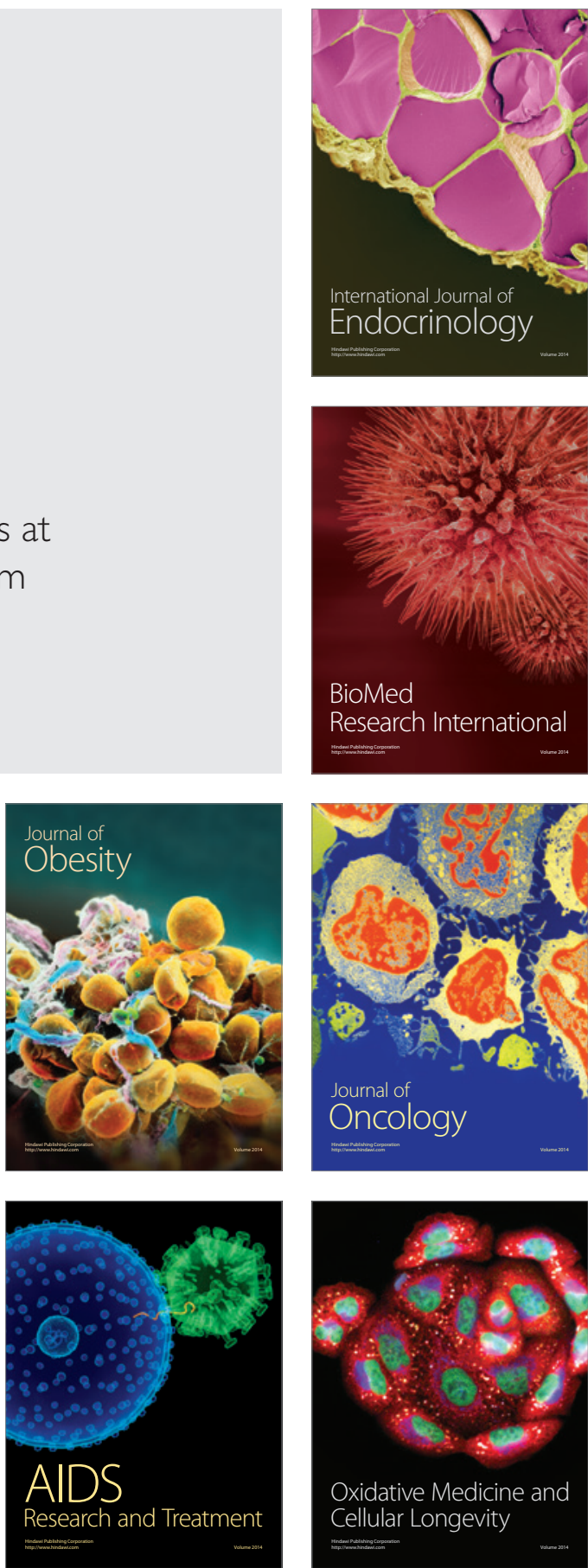